\title{
Contagem de carboidratos, estado nutricional e perfil metabólico em adolescentes com diabetes mellitus tipo 1
}

\author{
Carbohydrate counting, nutritional status and metabolic profile of adolescents \\ with type 1 diabetes mellitus
}

\author{
Izabela Zibetti de Albuquerque ${ }^{1}$, Maria Luiza Ferreira Stringhini², Rosana de Morais Borges Marques ${ }^{3}$, \\ Cláudia Aparecida Mundim4, Monike Lourenço Dias Rodrigues ${ }^{5}$, Maria Raquel Hidalgo Campos ${ }^{6}$ \\ 1 Mestre em Nutrição e Saúde. Universidade Federal de Goiás, Hospital das Clínicas. Goiânia, GO, Brasil. \\ ${ }^{2}$ Doutora em Ciência Animal, Universidade Federal de Goiás, Faculdade de Nutrição. Goiânia, GO, Brasil. \\ 3 Doutora em Ciências da Saúde, Universidade Federal de Goiás, Faculdade de Nutrição. Goiânia, GO, Brasil. \\ ${ }^{4}$ Médica Endocrinologista, Universidade Federal de Goiás, Hospital das Clínicas. Goiânia, GO, Brasil. \\ ${ }^{5}$ Doutora em Medicina (Endocrinologia Clínica), Universidade Federal de Goiás, Faculdade de Medicina. Goiânia, GO, Brasil. \\ ${ }^{6}$ Doutora em Medicina Tropical, Universidade Federal de Goiás, Faculdade de Nutrição. Goiânia, GO, Brasil.
}

Este artigo compõe a dissertação apresentada pela autora ao Programa de Pós-Graduação em Nutrição e Saúde da Faculdade de Nutrição da Universidade Federal de Goiás para obtenção do título de Mestre em Nutrição e Saúde. Agência de fomento: Coordenação de Aperfeiçoamento de Pessoal de Nível Superior (Capes).

\section{RESUMO}

Objetivos: Avaliar o efeito de um programa de contagem de carboidratos sobre parâmetros antropométricos, de composição corporal, bioquímicos e de consumo alimentar em adolescentes com diabetes mellitus tipo 1 em uso de insulina.

Métodos: Ensaio clínico randomizado que incluiu adolescentes entre 10 e 19 anos, em tratamento para diabetes mellitus tipo 1 com associação de insulinas de ação rápida (regular) e intermediária (Neutral Protamine Hagedorn). Os participantes receberam orientação nutricional e foram acompanhados durante quatro meses, sendo divididos em grupo intervenção (GI), com contagem de carboidratos, e grupo controle (GC), sem contagem de carboidratos. No início e no final do programa os pacientes foram avaliados em relação ao índice de massa corporal, circunferência da cintura, percentual de gordura corporal e parâmetros bioquímicos: hemoglobina glicada, glicemia de jejum, glicemia pósprandial e perfil lipídico. O consumo alimentar foi avaliado a cada quinze dias por meio do recordatório de 24 horas. Para a comparação intragrupo foram utilizadosos testes T-pareado e Wilcoxon e para a comparação intergrupo os testes $t$ Student e Mann-Whitney. O nível de significância adotado foi o de $5 \%$.

Resultados: Participaram do estudo 28 adolescentes, $14 \mathrm{em}$ cada grupo. O GI diminuiu significativamente a hemoglobina glicada ( $\mathrm{p}=0,002)$ e a ingestão de lipídeos ( $\mathrm{p}=0,002)$, e aumentou a ingestão de carboidratos $(\mathrm{p}=0,005)$. O GC aumentou a hemoglobina glicada ( $\mathrm{p}=0,024)$. O GI apresentou menor valor de glicemia de jejum $(\mathrm{p}=0,033)$ e da hemoglobina glicada $(\mathrm{p}<0,001)$ em relação ao GC. Ambos os grupos diminuíram a ingestão calórica. Os parâmetros antropométricos permaneceram estáveis e não houve diferença entre os grupos.

Conclusões: A contagem de carboidratos associada ao uso das insulinas Neutral Protamine Hagedorn e Regular favoreceu o controle glicêmico, permitiu flexibilidade na ingestão de carboidratos, diminuiu a ingestão calórica e não influenciou na composição corporal.

DESCRITORES: DIABETES MELLITUS TIPO 1; HEMOGLOBINA A GLICOSILADA; ADOLESCENTE; CARBOIDRATOS DA DIETA; ESTADO NUTRICIONAL.

\section{ABSTRACT}

Aims: To evaluate the effect of a carbohydrate counting program on the anthropometric measurements, body composition, biochemical parameters anddietary intake of adolescentswithtype 1 diabetes mellitususing insulin.

Methods: A randomized clinical trial included adolescents between 10 and 19 years in treatment for type 1 diabetes with association of fastacting insulin (regular) and intermediate-acting insulin (Neutral Protamine Hagedorn). The participants received nutritional counseling and were followed for four months, being divided into intervention group (IG), with carbohydrate counting, and control group (CG), without carbohydrate counting. At the beginning and end of the program, patients were evaluated for body mass index, waist circumference, body fat percentage and biochemical parameters: glycated hemoglobin, fasting glucose, postprandial glucose and lipid levels. Dietary intake was assessed every two weeks through the 24-hour recall. For intragroup comparison T-paired and Wilcoxon tests were used, and for intergroup comparison Student $t$ and Mann-Whitney tests were used. The significance level was 5\%.

Results: The study included 28 adolescents, 14 in each group. The IG significantly decreased glycated hemoglobin ( $\mathrm{p}=0.002)$ and lipid intake $(p=0.002)$, and increased carbohydrate intake $(p=0.005)$. The CG increased glycated hemoglobin $(p=0.024)$. The IG showed lower fasting glucose value $(\mathrm{p}=0.033)$ and glycated hemoglobin $(\mathrm{p}<0.001)$ compared to the CG. Both groups decreased caloric intake. Anthropometric parameters were stable and there was no difference between groups.

Conclusions: Carbohydrate counting together with Neutral Protamine Hagedorn and Regular insulins favored glycemic control, allowed flexibility in carbohydrate intake, decreased caloric intake, and had no effect on body composition.

KEY WORDS: DIABETES MELLITUS, TYPE 1; HEMOGLOBIN A, GLYCOSYLATE; ADOLESCENT; DIETARY CARBOHYDRATES; NUTRITIONAL STATUS. 


\section{INTRODUÇÃO}

O diabetes mellitus tipo 1 (DM1) é um distúrbio metabólico consequente à destruição gradual das células beta pancreáticas produtoras de insulina, evoluindo para a deficiência total na secreção deste hormônio. ${ }^{1}$ Quando detectado na infância, a hiperglicemia prolongada pode comprometer precocemente a qualidade de vida, pelos danos vasculares que altas concentrações glicêmicas podem causar. $^{2}$

Resultados publicados pelo Diabetes Control and Complications Trial (DCCT) ${ }^{3}$ evidenciaram a importância do controle glicêmico adequado para retardar o início e a progressão de complicações micro e macrovasculares do DM1. Uma das principais recomendações desse estudo em relação aos aspectos nutricionais de indivíduos com DM1 foi de que o planejamento dietético deve ser individualizado, permitir flexibilidade nas escolhas alimentares e, sobretudo, possibilitar um controle metabólico adequado. ${ }^{3}$ Uma maneira de otimizar esse controle é ajustar o número de unidades de insulina administradas à quantidade de carboidratos consumidos, por meio da contagem de carboidratos da dieta. Nessa estratégia, o requerimento insulínico é proporcional ao conteúdo de carboidratos de um alimento ou refeição, visto que a glicemia pós-prandial é determinada pela quantidade consumida desse macronutriente. ${ }^{4}$

Embora os estudos apontem para a efetividade da contagem de carboidratos na melhora do controle glicêmico, ${ }^{5-7}$ especula-se sobre o ganho de peso decorrente do ajuste da dose de insulina ${ }^{8}$ e sua influência no perfil lipídico ${ }^{5,7}$ e no consumo alimentar., ${ }^{9,10}$ Em relação ao consumo alimentar, sugere-se que indivíduos praticantes da contagem de carboidratos prefiram alimentos gordurosos em detrimento às fontes de carboidratos. Diante do exposto, este estudo teve como objetivo avaliar o efeito da contagem de carboidratos sobre parâmetros antropométricos, de composição corporal, bioquímicos e de consumo alimentar em adolescentes com DM1.

\section{MÉTODOS}

Foi conduzido um ensaio clínico randomizado no Ambulatório de Endocrinologia do Hospital das Clínicas da Universidade Federal de Goiás (HC/UFG), durante o período de dezembro de 2010 a janeiro de 2012, com pacientes que estavam em acompanhamento neste Serviço. O estudo foi aprovado pelo Comitê de Ética em Pesquisa do HC/UFG sob parecer $n^{\circ}$ $153 / 2010$. Os pais ou responsáveis ou o próprio entrevistado, quando maior de 18 anos, assinaram o termo de consentimento, após serem esclarecidos sobre a pesquisa.

Os critérios de inclusão foram: idade entre 10 e 19 anos, ${ }^{11}$ diagnóstico clínico de DM1, utilizar a associação de insulinas de ação rápida (regular) e intermediária (Neutral Protamine Hagedorn - NPH), não ter doença celíaca e não ser gestante. Foram excluídos os pacientes que não aceitaram participar do estudo e os que não completaram o período de acompanhamento.

Durante quatro meses, os participantes receberam orientação nutricional e foram acompanhados, sendo distribuídos em dois grupos: grupo intervenção (GI), com contagem de carboidratos; e grupo controle (GC), sem contagem de carboidratos. A triagem foi realizada às segundas-feiras, no turno vespertino, período reservado para atender os pacientes com DM1. Aqueles que atendiam aos critérios de inclusão eram convidados a participar do estudo e sorteados para um dos grupos, conforme a ordem de chegada ao Ambulatório. A partir da decisão dos pesquisadores de que o primeiro paciente que atendesse aos critérios de inclusão entraria no GI, o segundo foi convidado a entrar no GC. Desta forma, foi feita a distribuição aleatória.

Os pacientes de ambos os grupos foram avaliados no início e ao final do período de acompanhamento. A coleta dos dados iniciais foi realizada na primeira consulta após a inclusão no estudo, utilizando um formulário estruturado que contemplava dados demográficos, socioeconômicos e clínicos (idade, sexo, escolaridade, renda, tempo de diagnóstico, insulinoterapia e estágio puberal); dados antropométricos e de composição corporal; parâmetros bioquímicos; e informações sobre consumo alimentar. A classificação do estágio puberal foi realizada pela equipe médica, a partir dos critérios de Tanner.

Para o GI foi prescrito um plano alimentar baseado na contagem de carboidratos, considerando os hábitos alimentares do indivíduo e o total de carboidratos habitualmente consumido por refeição. A partir do cálculo das necessidades diárias e dos dados colhidos na anamnese do paciente, os macronutrientes foram distribuídos no plano alimentar de acordo com as recomendações da American Diabetes Association $(\mathrm{ADA})^{2}$ e foi entregue a lista de substituição de alimentos proposta pelo Guia Alimentar para a População Brasileira. ${ }^{13}$ A razão insulina-carboidratos foi calculada a partir da regra dos 500 , que consiste em dividir este número pelo total de unidades de insulina aplicadas ao dia. Desta forma, obtém-se o quanto uma unidade de insulina metaboliza determinada 
quantidade de carboidratos consumidos. ${ }^{1}$ A partir disso, foi calculado o bolus alimentar, que é a dose de insulina necessária para cobrir os gramas de carboidratos. Além do bolus alimentar, quando as metas glicêmicas préprandiais estabelecidas pelo médico não eram atingidas, estimava-se o bolus correção. Para isso, calculava-se o Fator de Sensibilidade (1500 dividido pelo total de insulina/dia), o qual era dividido pela diferença entre a meta glicêmica e a glicemia do momento. ${ }^{1}$

Os pacientes do GI receberam um manual de contagem de carboidratos e um formulário para anotação diária dos alimentos consumidos nas grandes refeições (café da manhã, almoço e jantar), da quantidade de insulina aplicada como bolus alimentar e bolus correção e das glicemias pré e pós-prandiais, sendo esta última realizada duas horas após o início da refeição. Foi aplicado o método de contagem em gramas de carboidratos, que consiste em somar os gramas de carboidrato de cada alimento por refeição, obtendo-se informações em tabelas e manuais especializados. O GI foi acompanhado quinzenalmente, durante os quatro meses, por meio de visitas domiciliares realizadas por uma nutricionista. Em cada visita era aplicado um recordatório de 24 horas (R24h) e avaliado o registro de alimentos, insulina e monitorações glicêmicas dos 15 dias anteriores, instrumento utilizado para ajustar a razão insulina/carboidratos.

Para o GC foi prescrito plano alimentar e entregue a lista de substituição de alimentos, ${ }^{13}$ sem aplicar a contagem de carboidratos. O planejamento dietético também seguiu as recomendações para macronutrientes da ADA. ${ }^{2}$ Esses pacientesforam acompanhados a cada 15 dias, durante quatro meses, por telefone, quando a nutricionista coletava o R24h. Essa metodologia, que é validada, ${ }^{14}$ foi adotada para que o pesquisador não influenciasse o grupo controle com informações que extrapolassem as já recebidas no primeiro atendimento, realizado presencialmente. Para ambos os grupos foram apresentadas medidas caseiras padronizadas pelo Guia Alimentar para a População Brasileira ${ }^{13}$ para facilitar o relato quantitativo do consumo alimentar.

Para avaliar a fidedignidade das informações de consumo alimentar coletadas por métodos diferentes, presencial e por telefone, foi aferida a correlação entre ingestão calórica (IC) e taxa de metabolismo basal (TMB) para os dois grupos. A TMB foi calculada por meio das equações de Schofield, ${ }^{15}$ propostas pela Organização Mundial da Saúde. ${ }^{16}$ A relação IC/TMB foi utilizada como parâmetro de super e subestimação do autorrelato da ingestão calórica, de acordo com os seguintes pontos de corte: $<1,14$ (subestimação grave); 1,14-1,35 (subestimação); $\geq 2,4$ (superestimação). ${ }^{17,18}$
Os pacientes do GI e do GC foram orientados a monitorar a glicemia pré e pós-prandial de uma refeição do dia. A monitoração glicêmica era alternada, sendo um dia desjejum, outro dia almoço e, no outro, jantar. Esse procedimento se deu do início ao final do estudo, porém a refeição avaliada no dia nem sempre era a mesma para todos os pacientes.

Os dados antropométricos foram aferidos na primeira consulta e após os quatro meses de acompanhamento, pelo mesmo pesquisador, em duplicata, e calculadas as médias. As dobras cutâneas tricipital (DCT) e subescapular (DCSE) foram aferidas em triplicata e obtidas as medianas. Para aferir o peso, foi utilizada balança portátil tipo plataforma, digital e eletrônica, marca Tanita ${ }^{\circledR}$, com capacidade máxima de 150 quilos e sensibilidade de 50 gramas. A estatura foi medida com um estadiômetro portátil da marca Seca (modelo 208), com variação de $1 \mathrm{~mm}$ e extensão máxima de $220 \mathrm{~cm}$, fixado à parede sem rodapé. A circunferência da cintura (CC) foi medida com fita métrica inextensível, com comprimento máximo de $150 \mathrm{~cm}$ e variação de $1 \mathrm{~mm}$. Esses dados foram coletados segundo o proposto por Gibson. ${ }^{19}$ As dobras DCT e DCSE foram mensuradas com adipômetro Lange ${ }^{\circledR}$.

Foi calculado índice de massa corporal (IMC) dividindo o peso pela altura ao quadrado e o mesmo foi avaliado segundo a idade (IMC/I). ${ }^{20}$ A classificação desse índice e seu ponto de corte foram expressos em percentil, de acordo com o proposto pelo Sistema de Vigilância Alimentar e Nutricional (SISVAN). ${ }^{21}$

Os dados de CC foram avaliados por meio dos pontos de corte propostos por Mccarthy et al.,22 que considera normais os valores entre os percentis 10 e 90.A porcentagem de gordura corporal foi obtida com a soma das dobras DCT e DCSE utilizando-se a fórmula de Slaughter et al. ${ }^{23}$ e classificada em percentil segundo o National Center for Health Statistics (NCHS), ${ }^{24}$ considerando como normais os valores entre os percentis 15 e 90 .

Para a avaliação bioquímica foram determinadas as concentrações de hemoglobina glicada (A1C) pelos métodos Cromatografia Iônica, Trivelli Modificado, Cromatografia Líquida de Alta Performance e Turbidimetria, reconhecidos pelo National Glycohemoglobin Standardization Program (NGSP) e indicados pela $\mathrm{ADA}^{2}$ e pela Sociedade Brasileira de Diabetes (SBD). ${ }^{1}$ A glicemia de jejum (GJ) e a glicemia pós-prandial(GPP) foram avaliadas segundo a idade, conforme as mesmas diretrizes, assim como o perfil lipídico: colesterol total (CT) triglicerídeos (TG), colesterol ligado à lipoproteína 
de alta densidade (HDL-c) e colesterol ligado à lipoproteína de baixa densidade (LDL-c). A avaliação bioquímica foi realizada no início e após os quatro meses de acompanhamento.

Para a avaliação do consumo alimentar foram aplicados oito R24h em intervalos quinzenais. A composição nutricional das dietas ingeridas foi calculada em planilha do Excel que compila informações nutricionais de diversas tabelas de composição de alimentos, utilizada por Marques et al. ${ }^{25}$ e Teles e Fornés. ${ }^{26}$ A adequação do consumo de macronutrientes foi avaliada com base nas recomendações da ADA: ${ }^{2}$ carboidratos $45-65 \%$ do valor energético total (VET), lipídeos 25-35\% do VET, ácidos graxos saturados $\leq 7$, proteínas $10-20 \%$ do VET e fibras $14 \mathrm{~g} / 1000 \mathrm{kcal}$.

Para a elaboração do plano alimentar dos pacientes foram calculadas as necessidades estimadas de energia (NEE) no momento inicial do estudo. Para obter a NEE foi avaliado o nível de atividade física (NAF) de cada indivíduo. A prática de atividades diárias foi registrada em formulário dividido em 48 períodos de 30 minutos. Segundo o Institute of Medicine, ${ }^{27}$ cada atividade física tem um coeficiente de acordo com a idade e o gênero. Para determinar NAF foi realizada a soma dos coeficientes de atividade física e posterior divisão pelo total de períodos.

A classificação do NAF foi determinada por meio dos intervalos, sendo sedentário quando maior ou igual a 1,0 e menor que 1,4 ; leve quando maior ou igual a 1,4 e menor que 1,6 ; moderado quando maior ou igual a 1,6 e menor que 1,9 ; e intenso quando maior ou igual a 1,9 e menor que 2,5.27 Para o cálculo da NEE foram utilizadas as equações para adolescentes, por gênero. ${ }^{27}$

Por meio do aplicativo Gpower 3.1.2 obteve-se o número amostral mínimo de 28 pacientes, para utilização de teste t-pareado. Considerou-se nível de significância de $5 \%$, poder de teste de $80 \%$ e tamanho de efeito igual a 0,55 segundo a classificação de Cohen (1988). ${ }^{12}$ Os dados foram analisados pelo programa Statistical Package for the Social Sciences (SPSS, versão 18.0). Os resultados estão expressos em frequência e média (desvio padrão). Para as variáveis categóricas aplicou-se o teste Qui-Quadrado ou teste Exato de Fisher. Foi avaliada a distribuição das variáveis contínuas por meio do teste Shapiro Wilk, indicado para amostras com $n \leq 30$. Para a comparação intragrupo foi utilizado o teste T-pareado para as variáveis paramétricas e teste de Wilcoxon para as não paramétricas. Para a comparação intergrupo, referente aos deltas, foram utilizados os testes $t$ Student para variáveis com distribuição simétrica e Mann-Whitney para aquelas com distribuição assimétrica. O nível de significância adotado foi o de 5\%.

\section{RESULTADOS}

Dos 41 indivíduos que preencheram os critérios de inclusão no período do estudo, 20 foram alocados no GI e 21 no GC. Houve seis perdas no GI (três pacientes não consentiram em participar do estudo e três foram excluídos por não seguirem o protocolo de acompanhamento proposto). No grupo controle, houve perda de sete pacientes, todas por falta de adesão ao tratamento. Dessa forma, para as análises, cada grupo foi composto por 14 pacientes. As perdas se manifestaram na metade do estudo.

Dos 28 adolescentes avaliados, $16(57,1 \%)$ eram do sexo feminino, 21 (75\%) tinham idade inferior a 15 anos e $21(75 \%)$ cursavam, no momento inicial do estudo, o ensino fundamental. A renda per capita de 25 pacientes $(89,2 \%)$ foi inferior a um saláriomínimo, caracterizando uma população de baixo poder aquisitivo.

Em relação às características clínicas iniciais, 14 adolescentes (50\%) apresentaram tempo de duração da doença inferior a cinco anos e $15(53,5 \%)$ foram classificados em pré-púberes de acordo com o estágio puberal. A dose média de insulina por quilograma de peso corporal aplicada ao dia (U/ $\mathrm{kg} / \mathrm{dia})$ foi de $0,71 \pm 0,29$ e $0,90 \pm 0,31$ para o GI e GC, respectivamente. Quanto à classificação do nível de atividade física, 21 pacientes $(75 \%)$ eram sedentários ou desempenhavam atividades leves. Não houve diferenças $(p>0,05)$ entre os grupos em relação a essas variáveis no início do estudo.

Houve prevalência de excesso de peso em 17,8\% dos indivíduos do estudo. Oito pacientes $(28,5 \%)$ apresentaram valores de circunferência da cintura acima do percentil desejável (percentil 85) e todos se encontravam com percentuais de gordura corporal dentro da normalidade.

Ao início do estudo, as características bioquímicas foram semelhantes entre os dois grupos. O percentual de inadequação da A1C foi de 39,3\% para o GI e 28,6\% para o GC, sem haver diferença significativa entre os grupos $(\mathrm{p}>0,05)$.

$\mathrm{Na}$ Tabela 1 observa-se que os grupos, inicialmente, não se diferenciaram em relação aos parâmetros antropométricos, de composição corporal, bioquímicos e de consumo alimentar $(\mathrm{p}>0,05)$. Assim, foram considerados homogêneos no momento inicial do estudo. 
Tabela 1. Parâmetros antropométricos, composição corporal, bioquímicos e de consumo alimentar de adolescentes portadores de diabetes mellitus tipo 1 dos grupos controle e intervenção antes do início do estudo

\begin{tabular}{|lccc}
\multicolumn{1}{c}{ Variáveis } & $\begin{array}{c}\text { Grupo Controle } \\
(\mathbf{n = 1 4 )} \\
\text { Média (desvio padrão) }\end{array}$ & $\begin{array}{c}\text { Grupo Intervenção } \\
(\mathbf{n = 1 4 )} \\
\text { Média (desvio padrão) }\end{array}$ & p \\
\hline IMC (kg/m²) & $19,08(2,67)$ & $20,40(2,80)$ & $0,178^{+}$ \\
\hline Circunferência da cintura (cm) & $65,53(6,05)$ & $68,76(5,63)$ & $0,156^{*}$ \\
\hline Gordura corporal (\%) & $25,32(6,05)$ & $29,42(9,05)$ & $0,334^{+}$ \\
\hline Glicemia de jejum (mg/dL) & $218,78(134,18)$ & $217,92(92,58)$ & $0,676^{+}$ \\
\hline Glicemia pós-prandial (mg/dL) & $257,40(168,35)$ & $257,45(168,21)$ & $0,999^{*}$ \\
\hline A1C (\%) & $8,42(2,14)$ & $10,59(3,43)$ & $0,091^{+}$ \\
\hline Colesterol total (md/dL) & $177,23(34,12)$ & $163,11(36,25)$ & $0,326^{*}$ \\
\hline Triglicerídeos (md/dL) & $73,23(41,61)$ & $91,83(51,74)$ & $0,313^{+}$ \\
\hline HDL-colesterol (md/dL) & $50,77(13,92)$ & $49,66(9,65)$ & $0,822^{*}$ \\
\hline LDL-colesterol (md/dL) & $111,75(28,91)$ & $95,28(30,65)$ & $0,113^{+}$ \\
\hline NEE (kcal) & $2129,20(558,13)$ & $2006,41(448,99)$ & $0,603^{+}$ \\
\hline Consumo calórico total (kcal) & $1762,84(604,17)$ & $1844,60(805,08)$ & $0,701^{+}$ \\
\hline kcal de carboidratos (\%) & $50,28(11,03)$ & $44,02(9,09)$ & $0,194^{+}$ \\
\hline kcal de proteínas (\%) & $20,73(4,77)$ & $21,66(4,77)$ & $0,667^{+}$ \\
\hline kcal de lipídeos (\%) & $28,98(9,70)$ & $34,31(7,89)$ & $0,114^{+}$ \\
\hline kcal de gordura saturada (\%) & $9,03(2,45)$ & $10,05(2,51)$ & $0,376^{+}$ \\
\hline Fibras (g/1000 kcal) & $15,26(3,81)$ & $13,59(6,84)$ & $0,150^{+}$ \\
\hline
\end{tabular}

* Teste $t$ Student; ${ }^{\dagger}$ Teste de Mann-Whitney; IMC: índice de massa corporal; A1C: hemoglobina glicada; NEE: necessidade estimada de energia.
Na comparação intragrupo (valor de $\mathrm{p}$ representado por $\mathrm{p}^{1}$ e $\mathrm{p}^{2}$ ), correspondente aos quatro meses de acompanhamento (momento inicial e final), o $\mathrm{GC}$ não apresentou diferença $(\mathrm{p}>0,05)$ em relação às variáveis antropométricas e de composição corporal. Para os parâmetros bioquímicos, aumentou de forma significativa a A1C $(p=0,024)$. Em relação ao consumo alimentar, houve redução significativa apenas no consumo calórico total $(p=0,005)$ (Tabela 2).

Nesta mesma avaliação, do início até o final dos quatro meses, o GI não apresentou diferença $(p>0,05)$ entre o IMC, a CC e a gordura corporal. A GJ e a GPP também não se alteraram, porém, foi encontrada redução significativa na $\mathrm{A} 1 \mathrm{C}(-2,20 \pm 2,31 ; \mathrm{p}=0,002)$. Embora a dose média de insulina aplicada ao dia tenha aumentado de $0,90 \pm 0,31$ para $1,01 \pm 0,35 \mathrm{U} / \mathrm{kg} / \mathrm{dia}$, este aumento não foi significativo (Tabela 2).

Entre o início e o final do programa, houve aumento das concentrações de CT e LDL-c ( $p=0,009$ e p=0,012, respectivamente) no grupo intervenção, entretanto, não houve diferença significativa entre a porcentagem de pacientes que estavam dentro do alvo lipídico de CT ao início do estudo ( $\mathrm{n}=10,71,4 \%)$, e os que se mantiveram nos limites de normalidade ao final dos quatro meses ( $n=9,64,3 \%)$. Para o LDL-c também não foi observada diferença $(p>0,05)$ entre as porcentagens de adequação no início e final do estudo. Nenhuma alteração significativa foi encontrada no HDL-c e nos TG (Tabela 2).

No tocante ao consumo alimentar do GI, reduções significativas foram observadas no consumo calórico total $(p=0,002)$ e na porcentagem de lipídeos em relação às calorias totais ingeridas $(p=0,002)$. $O$ consumo relativo de carboidratos aumentou significativamente $(\mathrm{p}=0,005) \mathrm{e}$, para as proteínas, gordura saturada e fibras, não foram encontradas alterações significativas (Tabela 2). Tanto o GC quanto o GI apresentaram subestimação grave do auto relato da ingestão calórica. A relação média de IC/TMB foi de 0,98 para o GC e 0,89 para o GI.

Na comparação intergrupo (valor de p representado por $\mathrm{p}^{3}$ ), GJ, A1C, CT e LDL-c apresentaram diferenças significativas entre GI e GC. Em comparação ao GC, o GI apresentou maior redução do valor de GJ quando avaliado pelo delta $(p=0,033)$, assim como da $\mathrm{A} 1 \mathrm{C}(\mathrm{p}<0,001)$, e incremento na concentração de CT $(p=0,004)$ e LDL-c $(p=0,019)$. Para os demais parâmetros bioquímicos, antropométricos, de composição corporal e de consumo alimentar não houve diferença entre os grupos $(\mathrm{p}>0,05)$.

Não foram identificadas diferenças significativas das glicemias médias pré e pós-prandiais comparadas intragrupo e intergrupo durante os quatro meses de acompanhamento (Figura 1). Porém, observou-se tendência de diferença entre a média glicêmica préalmoço dos grupos $(\mathrm{p}=0,075)$. 
Tabela 2. Média e desvio-padrão dos parâmetros antropométricos, de composição corporal, bioquímicos e de consumo alimentar apresentados pelos adolescentes portadores de diabetesmellitus tipo 1 ao início e ao final do estudo e comparação entre os dois grupos em relação às diferenças obtidasentre os dois momentos

\begin{tabular}{|c|c|c|c|c|c|c|c|c|c|}
\hline \multirow{2}{*}{ Variáveis } & \multicolumn{2}{|c|}{ Grupo Controle } & \multirow{2}{*}{$\mathbf{p}^{1}$} & \multirow{2}{*}{$\begin{array}{l}\text { Delta (valor final- } \\
\text { valor inicial) }\end{array}$} & \multicolumn{2}{|c|}{ Grupo Intervenção } & \multirow{2}{*}{$\mathrm{p}^{2}$} & \multirow{2}{*}{$\begin{array}{l}\text { Delta (valor final- } \\
\text { valor inicial) }\end{array}$} & \multirow{2}{*}{$p^{3}$} \\
\hline & Início & Final & & & Início & Final & & & \\
\hline $\mathrm{IMC}\left(\mathrm{kg} / \mathrm{m}^{2}\right)$ & $19,08(2,67)$ & $19,20(2,91)$ & $1,000^{+}$ & $0,11(1,07)$ & $20,40(2,80)$ & $21,00(2,75)$ & $0,086^{*}$ & $0,60(1,20)$ & $0,269^{\ddagger}$ \\
\hline Circunferência da cintura $(\mathrm{cm})$ & $65,53(6,05)$ & $65,96(6,06)$ & $0,473^{*}$ & $0,42(2,60)$ & $68,76(5,63)$ & $69,72(5,92)$ & $0,203^{*}$ & $0,96(2,70)$ & $0,596^{\ddagger}$ \\
\hline Gordura corporal (\%) & $25,32(6,05)$ & $25,77(6,54)$ & $0,502^{+}$ & $0,44(2,88)$ & $29,42(9,05)$ & $31,89(10,20)$ & $0,093^{+}$ & $-3,82(4,40)$ & $0,312^{\ddagger}$ \\
\hline Glicemia de jejum (mg/dL) & $218,78(134,18)$ & $214,15(89,09)$ & $0,080^{+}$ & $15,00(138,81)$ & $217,92(92,58)$ & $158,71(78,38)$ & $0,054^{*}$ & $-59,21(104,35)$ & $0,033^{\ddagger}$ \\
\hline Glicemia pós-prandial (mg/dL) ${ }^{a}$ & $257,40(168,35)$ & $350,00(90,37)$ & $0,297^{+}$ & $62,71(137,56)$ & $276,88(181,61)$ & $290,11(179,40)$ & $0,889^{*}$ & $13,22(276,03)$ & $0,672^{\ddagger}$ \\
\hline $\mathrm{A} 1 \mathrm{C}(\%)$ & $8,42(2,14)$ & $9,62(2,91)$ & $0, \mathbf{0 2 4}^{+}$ & $1,26(2,11)$ & $10,59(3,43)$ & $8,39(2,28)$ & $0,002^{+}$ & $-2,20(2,31)$ & $<0,001^{\text {s }}$ \\
\hline Colesterol total $(\mathrm{mg} / \mathrm{dL})^{\mathrm{b}}$ & $181,33(36,90)$ & $179,00(39,19)$ & $0,720^{*}$ & $-2,33(18,84)$ & $166,30(36,21)$ & $194,09(54,09)$ & $0,009^{*}$ & $27,78(28,33)$ & $0,004^{\S}$ \\
\hline Triglicerídeos (mg/dL) & $73,23(41,61)$ & $79,20(31,08)$ & $0,164^{+}$ & $13,77(23,04)$ & $91,83(51,74)$ & $128,00(144,73)$ & $0,278^{+}$ & $44,54(116,15)$ & $0,956^{\varsigma}$ \\
\hline HDL-colesterol $(\mathrm{mg} / \mathrm{dL})^{c}$ & $46,44(13,38)$ & $44,33(14,73)$ & $0,359^{*}$ & $-2,11(6,54)$ & $50,00(10,04)$ & $54,45(11,93)$ & $0,247^{*}$ & $4,45(12,02)$ & $0,160^{\ddagger}$ \\
\hline LDL-colesterol $(\mathrm{mg} / \mathrm{dL})^{\mathrm{d}}$ & $121,08(28,92)$ & $118,62(30,50)$ & $0,660^{*}$ & $-2,46(16,21)$ & $95,28(30,65)$ & $114,25(40,48)$ & $0,012^{+}$ & $21,56(23,05)$ & $0,019^{\ddagger}$ \\
\hline Consumo calórico total (kcal) & $1762,84(604,17)$ & $1365,63(317,76)$ & $0,005^{+}$ & $-397,20(473,82)$ & $1844,60(805,08)$ & $1301,88(427,27)$ & $0,002^{+}$ & $-542,71(524,09)$ & $0,571^{\mathrm{s}}$ \\
\hline kcal de carboidratos (\%) & $50,28(11,03)$ & $52,18(5,67)$ & $0,542^{+}$ & $1,90(12,39)$ & $44,02(9,09)$ & $51,14(3,52)$ & $0,005^{+}$ & $7,12(7,38)$ & $0,352^{\S}$ \\
\hline kcal de proteínas (\%) & $20,73(4,77)$ & $20,94(5,34)$ & $0,952^{+}$ & $0,20(6,07)$ & $21,66(4,77)$ & $21,16(3,51)$ & $0,903^{+}$ & $-0,49(5,35)$ & $0,910^{\S}$ \\
\hline kcal de lipídeos (\%) & $28,98(9,70)$ & $26,94(5,34)$ & $0,542^{+}$ & $-2,03(10,07)$ & $34,31(7,89)$ & $27,68(4,93)$ & $0,002^{+}$ & $-6,63(7,37)$ & $0,194^{\S}$ \\
\hline kcal de gordura saturada (\%) & $9,03(2,45)$ & $8,77(1,45)$ & $1,000^{+}$ & $-0,26(3,04)$ & $10,05(2,51)$ & $9,06(1,77)$ & $0,473^{+}$ & $-0,98(2,85)$ & $0,427^{\S}$ \\
\hline Fibras (g)/1000 kcal & $15,26(3,81)$ & $16,29(3,38)$ & $0,483^{*}$ & $1,02(5,32)$ & $13,59(6,84)$ & $14,06(4,31)$ & $0,761^{+}$ & $0,47(5,90)$ & $0,796^{\ddagger}$ \\
\hline
\end{tabular}

* Teste T-pareado; ${ }^{\dagger}$ Teste de Wilcoxon; ${ }^{\ddagger}$ Teste $t$ de Student; ${ }^{\S}$ Teste de Mann-Whitney; a 9 pacientes pareados no grupo intervenção; b,c 9 pacientes pareados no grupo controle e 11 no grupo intervenção; ${ }^{d} 9$ pacientes pareados no grupo controle; IMC: índice de massa corporal; A1C: hemoglobina glicada. $\mathrm{p}^{1}$ : comparação intragrupocontrole; $\mathrm{p}^{2}$ : Comparação intragrupo intervenção; $p^{3}$ comparaçãointergrupo referente aos deltas.

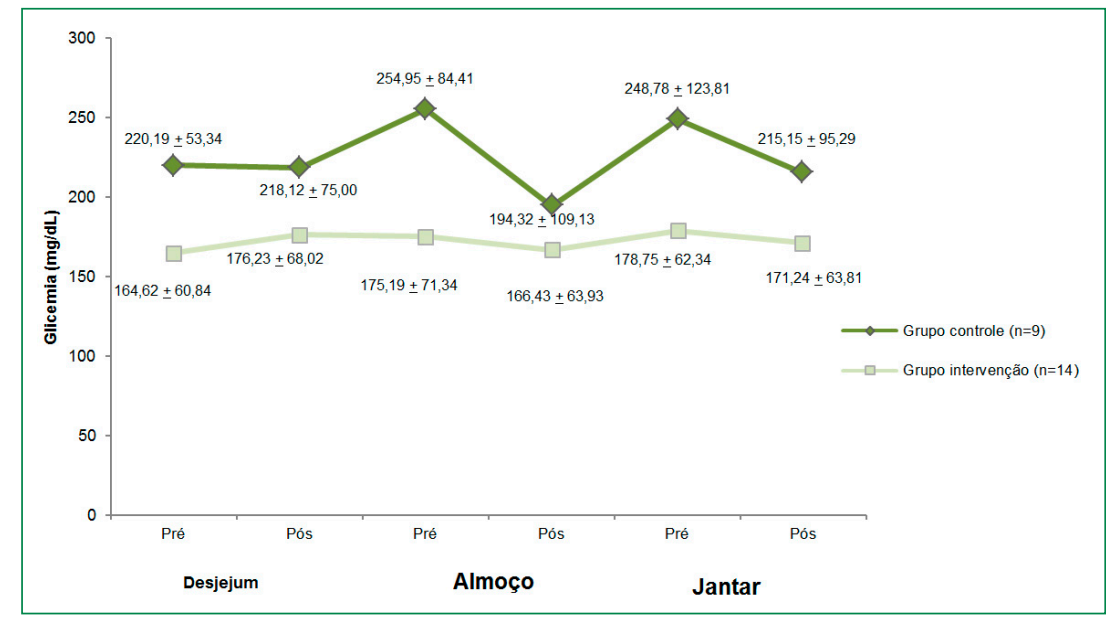

Figura 1. Média glicêmica pré e pós-prandial dos grupos controle e intervenção durante os quatro meses de acompanhamento

\section{DISCUSSÃO}

O plano alimentar de adolescentes com DM1 deve estar de acordo com as recomendações nutricionais de energia e nutrientes específicas para essa faixa etária, para proporcionar o crescimento e desenvolvimento adequados e, juntamente com a reposição insulínica, fornecer flexibilidade às suas atividades. ${ }^{1} \mathrm{~A}$ contagem de carboidratos é um método que visa o controle glicêmico e deve estar associada a um contexto de hábitos alimentares saudáveis. ${ }^{4}$ Neste estudo, verificamos que a intervenção por meio desse método diminuiu significativamente a GJ, a A1C e a ingestão calórica e de lipídeos, aumentou as concentrações de CT e de LDL-c e a ingestão de carboidratos, e não influenciou na composição corporal.

A melhora da $\mathrm{A} 1 \mathrm{C}$ e da GJ do grupo intervenção confirma a necessidade de adequação de insulina ao consumo de carboidratos da pessoa com DM1, principalmente por se tratar de uma doença crônica que, se mal controlada, pode comprometer precocemente a saúde do paciente. O U.K. Prospective Diabetes Study ${ }^{28}$ mostrou que a redução da A1C e das concentrações de GJ foram acompanhadas por 
diminuições substanciais nas complicações micro e macrovasculares do diabetes.

Neste estudo, a média de diminuição da A1C no grupo intervenção foi de $2,20 \pm 2,31$ pontos percentuais. A queda de 1\% neste parâmetro bioquímico reduz em $14 \%$ o risco de infarto do miocárdio e $37 \%$ o risco de complicações microvasculares. ${ }^{3,28}$

Além disso, os valores de A1C apresentados pelos pacientes em contagem de carboidratos ao início e ao final do estudo, se transformados para valores de glicemia média estimada (GME), ${ }^{1}$ correspondem a um valor inicial maior que $249 \mathrm{mg} / \mathrm{dL}$ e valor final inferior a $197 \mathrm{mg} / \mathrm{dL}$. Em contrapartida, a piora do controle glicêmico do GC se traduz em valor inicial de GME de $197 \mathrm{mg} / \mathrm{dL}$ e final de $226 \mathrm{mg} / \mathrm{dL}$.

Embora não tenha sido observada diferença significativa entre as médias pré e pós-prandial dos grupos (Figura 1), o GC apresenta maior oscilação glicêmica em contraste à constância das glicemias dos pacientes em contagem de carboidratos. Do ponto de vista clínico, a flutuação da glicemia é mais prejudicial para a função endotelial do que altos níveis glicêmicos constantes. ${ }^{29} \mathrm{Na}$ oscilação glicêmica as células não são capazes de aumentar suas defesas antioxidantes, condição que pode favorecer o desenvolvimento de complicações do diabetes. ${ }^{30,31}$

A subestimação grave da ingestão calórica autorrelatada pode ter favorecido a redução significativa do consumo calórico total observado em ambos os grupos. A aplicação repetitiva de recordatórios de 24 horas e o conhecimento do indivíduo sobre alimentação saudável são alguns fatores que podem levar à subnotificação do real consumo calórico total. O indivíduo tende a dar repostas "socialmente aceitáveis" que implicam no sub-relato do consumo calórico e, consequentemente, na omissão da ingestão de alimentos doces e gordurosos. ${ }^{32}$

No estudo de Helgeson et al. ${ }^{9}$ verificou-se que o consumo calórico de adolescentes com DM1 foi inferior ao recomendado, o que corrobora com nossos achados. Esses autores ressaltam que os estudos de validação de medidas de consumo alimentar têm apontado para a subestimação do consumo de calorias, independente da característica da população em que o inquérito dietético está sendo validado. Ainda nesse mesmo estudo, houve maior consumo relativo de gordura em detrimento aos carboidratos em comparação a adolescentes não diabéticos.

Overby et al. ${ }^{10}$ também encontraram, em adolescentes diabéticos, maior consumo calórico a partir de gorduras totais e gorduras saturadas. Sugere-se que adolescentes que aplicam a contagem de carboidratos tendem a preferir alimentos com alto teor de gorduras, numa atitude compensatória, pois para o consumo de lipídeos não são requeridas doses de insulina, embora a gordura prolongue a resposta glicêmica, provocando uma hiperglicemia pós-prandial tardia. ${ }^{33}$ Casuísticas recentes demonstram a associação do maior consumo dietético de gorduras com o aumento das concentrações de A1C..$^{34,35} \mathrm{O}$ maior consumo energético a partir de gordura tem apresentado relação direta com o aumento de IMC e CC em pacientes diabéticos tipo 1. Isso reforça a necessidade de que a contagem de carboidratos e a flexibilidade alimentar inerente ao método seja pautada em escolhas saudáveis. Neste estudo, assim como no de Magliono et al., ${ }^{36}$ os pacientes que aplicaram a contagem de carboidratos apresentaram melhora significativa nas concentrações de A1C, aumento no consumo de carboidratos e diminuição no consumo de gorduras e proteínas, sem que esse método de abordagem nutricional interferisse na distribuição da gordura corporal. Não há evidências suficientes para afirmar que a aplicação da contagem de carboidratos provoque mudanças antropométricas e de composição corporal, quer sejam positivas ou negativas.

Rabasa-Lhoret et al. ${ }^{37}$ mostraram que dietas com $55 \%$ de carboidratos não modificaram o controle glicêmico quando comparadas a dietas hipoglicídicas, com $40 \%$ de carboidratos, desde que o número de unidades de insulina estivesse ajustado adequadamente à quantidade de carboidrato consumido. Além do mais, dietas reduzidas em carboidratos têm mostrado relação com o aumento do IMC e das concentrações de A1C. ${ }^{38}$ Para que a razão insulina/carboidratos seja adequada, é importante levar em consideração a sensibilidade insulínica do paciente. É comum notar sensibilidade menor no período da manhã (fenômeno do alvorecer), estabelecendo razões diferentes pela manhã e à noite. ${ }^{1}$

Nesta casuística, houve aumento significativo de CT e LDL-c no GI. Contudo, mesmo com o aumento dessas frações lipídicas, os valores se mantiveram na faixa de normalidade preconizada pela $\mathrm{ADA}^{2}{ }^{2}$ e não houve diferença significativa entre o percentual de indivíduos que estavam dentro do alvo lipídico ao início e ao final do estudo.O consumo de proteínas e gorduras saturadas do GI esteve acima do recomendado ao início, e permaneceu ao final do acompanhamento. O conteúdo de gordura saturada influencia diretamente as concentrações lipídicas plasmáticas, em especial o colesterol sérico. A absorção de gordura saturada não é limitada, como ocorre com o colesterol dietético na luz intestinal, e, por isso, sua ingestão promove efeito mais intenso sobre a colesterolemia. ${ }^{39}$ 
Arcanjo et al. ${ }^{40}$ observaram que crianças e adolescentes com DM1 têm maior prevalência de CT e LDL-c alterados em comparação aos não diabéticos. A hiperglicemia crônica propicia maior oxidação de partículas de LDL-c, que se acumulam em partículas densas mais aterogênicas. ${ }^{41}$

Foi documentado por Queiroz et al. ${ }^{42}$ que variáveis inseridas no hábito de vida de crianças e adolescentes, como o consumo da merenda oferecida na escola ou o lanche adquirido nesse estabelecimento, tendem a afetar negativamente o controle glicêmico. Ao aprender sobre contagem de carboidratos, o paciente deve compreender que, apesar de diferentes alimentos conterem quantidades equivalentes de carboidratos, isso não significa que eles são igualmente saudáveis. A flexibilidade que esse método permite na escolha e na quantidade de alimentos que serão consumidos torna ainda mais imprescindível a educação nutricional em pacientes diabéticos.

Os resultados obtidos pelo presente estudo sugerem a possibilidade da associação das insulinas regular e NPH à contagem de carboidratos para melhora do controle glicêmico. Assim, recomenda-se que essa estratégia seja inserida ao protocolo de atendimento nutricional a pacientes com DM1, acompanhada de educação nutricional e em diabetes, reforçando continuamente ao paciente a importância do cumprimento das orientações do plano alimentar. A assistência nutricional deve estar integrada à terapêutica do DM1, pois o estado nutricional do paciente precisa ser continuamente acompanhado e avaliado, de forma que intervenções nutricionais direcionadas possam ser feitas e a educação alimentar e nutricional seja aplicada.
Como limitações do estudo, tem-se que o tempo de acompanhamento não foi suficiente para alcançar os valores de A1C recomendados pela ADA. É possível que um tempo maior pudesse controlar e estabilizar essas concentrações. Quanto à utilização de diferentes laboratórios, todos os métodos utilizados nesse estudo para a avaliação da hemoglobina glicada são reconhecidos pelo NGSP e indicados pela ADA e pela SBD. Esta última entidade, por exemplo, refere em suas Diretrizes, ${ }^{1}$ que " a dosagem de HbAlc pode ser realizada por diversos métodos, sendo o ideal a Cromatografia Líquida de Alta Performance (HPLC), e que, mesmo por diferentes métodos aceitos para dosagem, os resultados sejam calibrados de modo a ter a mesma faixa de referência, de acordo com a utilizada no DCCT".

A avaliação do consumo alimentar foi realizada apenas por inquéritos quantitativos, sendo que a utilização do Questionário de Frequência de Consumo Alimentar permitiria discussões mais profundas a respeito da qualidade dietética. Os achados deste estudo referem-se a uma parcela de pacientes que frequentam um ambulatório especializado em DM1, portanto estes dados não podem ser extrapolados e generalizados para uma população maior.

Apesar das limitações apontadas, este estudo possibilitou verificar que a contagem de carboidratos foi eficiente em melhorar o controle glicêmico sem prejudicar os parâmetros antropométricos. A flexibilidade na escolha dos alimentos que essa estratégia oferece permitiu maior consumo de carboidratos, diminuição do consumo de gordura e calorias totais e não influenciou na composição corporal.

\section{AGRADECIMENTOS}

Auxílio financeiro: Coordenação de Aperfeiçoamento de Pessoal de Nível Superior (Capes) por meio da concessão da bolsa de mestrado.

\section{REFERÊNCIAS}

1. Sociedade Brasileira de Diabetes. Diretrizes da Sociedade Brasileira de Diabetes 2013-2014. São Paulo: AC Farmacêutica; 2014.

2. American Diabetes Association. Standards of medical care in diabetes - 2010. A position Statement of the American Diabetes Association. Diabetes Care.2010;33 Suppl 1:S11-61.

3. The Diabetes Control and Complications Trial Research Group. The effect of intensive treatment of diabetes on development and progression of long-term complications in insulin-dependent diabetes mellitus. N Engl J Med.1993;329(14):977-86.

4. Sociedade Brasileira de Diabetes. Manual de Contagem de Carboidratos. São Paulo; 2009.

5. Dias VM, Pandini JA, Nunes RR, Sperandei SLM, Portella ES, Cobas RA, et al. Effect of the carbohydrate counting method on glycemic control in patients with type 1 diabetes. Diabetology\& Metabolic Syndrome.2010;2(54):1-7.

6. Trento M, Borgo E, Kucich C, Passera P, Trinetta A, Charrier L, et al. Quality of life, coping ability, and metabolic control in patients with type 1 diabetes managed by group care and a carbohydrate counting program. Diabetes Care.2009;32(11):134.

7. Scavone G, Manto A, Pitocco D, Gagliardi L, Caputo S, Mancini L, et al. Effect of carbohudrate counting and medical nutritional therapy on glycaemic control in type 1 diabetic subjects: a pilot study. Diabet Med.2010;27(4):477-9. 
8. Gillespie SJ, Kulkarni KD, Daly AE. Using carbohydrate counting in diabetes clinical practice. J Am Diet Assoc. 1998;98(8): 897-905.

9. Helgeson VS, Viccaro L, Becker D, Escobar O, Siminerio L. Diet of adolescents with and without diabetes: Trading candy for potato chips? Diabetes Care. 2006;29(5):982-7.

10. Overby NC, Margeirsdottir HD, Brunborg C, Andersen LF, Dahl-Jorgenses K. The influence of dietary intake and meal pattern on blood glucose control in children and adolescents using intensive insulin treatment. Diabetologia. 2007;50(10):2044-51.

11. Organização Pan-Americana de Saúde. La salud del adolescente y el joven en las Américas. Washington DC: OPS; 1985.

12. Cohen J. Statistical power analysis for the behavioral sciences. 2nd ed. Hillsdale, NJ: Erlbaum; 1988.

13. Brasil. Ministério da Saúde. Secretaria de Atenção à Saúde. Departamento de Atenção Básica. Coordenação Geral da Política de Alimentação e Nutrição. Guia alimentar para a população brasileira: promovendo a alimentação saudável. Brasília; 2006. 210 p.

14. Casey PH, Goolsby SL, Lensing SY, Perloff BP, Bogle ML. The use of telephone interview methodology to obtain 24-hour dietary recalls. JAMA. 1999;99(11):1406-11.

15. Schofield WN. Predicting basal metabolic rate, new standards and rewiew of previous work. Hum NutrClinNutr. 1985;39 (supp 1):5-41.

16. World Health Organization. Report of a Joint FAO/WHO/UNU Expert Consultation. Human energy requirements. Food and nutrition technical reports series. Geneva: WHO; 2001.

17. Goldberg GR, Black AE, Jebb AS, Cole TJ, Murgatroyd PR, Coward WA, et al. Critical evaluation of energy physiology: derivation of cut-offs limits to identify under-recording. Eur J ClinNutr. 1991;45:S69-81.

18. Johansson L, Solvoll K, Bjorneboe G-EAa, JornDrevon CA. Under-Under and overreporting of energy intake related to weight status and lifestyle in a nationwide sample. Am J ClinNutr. 1998;68:266-74.

19. Gibson RS. Principles of nutritional assessment. 2nd ed. Oxford: Oxford University Press; 2005.

20. de Onis M, Onyango AW, Borghi E, Siyam A, Nishida C, Siekmann J. Development of a WHO growth reference for school-aged children and adolescents. Bull World Health Organ. 2007;85(9):660-7.

21. Ministério da Saúde. Sistema de Vigilância Alimentar e Nutricional-SISVAN. Orientações para a coleta e análise de dados antropométricos em serviços de saúde. Norma técnica. Material preliminar. Brasil, 2008.

22. Mccarthy HD, Jarret KV, Crawley HF. The development of waist circumference percentiles in British child age 5,0-16,9. Eur J ClinNutr. 2001;55(10):902-7.

23. Slaughter M, Lohman T, Boileau R. Human Biol. 1988;60:709-23.

24. National Center For Health Statistics (NCHS). Vital and Health Statistics11(238).AnthropometricReference Data andPrevalence ofOverweight. United States, 1976-80. US Department of Health and Human Services:Hyattsville;1987.

25. Marques RMB, Fornés NS, Stringhini MLF. Fatores socioeconômicos, demográficos, nutricionais e de atividade física de adolescentes portadores de diabetes melito tipo 1. Arq Bras EndocrinolMetab. 2011;55(3):194-202.

26. Teles SAS, Fornés NS. Consumo alimentar e controle metabólico em crianças e adolescentes portadores de diabetes melito tipo 1. Rev Paul Pediatr. 2011;29(3):378-84.

27. Institute of Medicine. Dietary reference intakes for energy, carbohydrate, fiber, fat, fatty acids, cholesterol, protein and aminoacids. Washington: IOM; 2005.

28. U. K. Prospective Diabetes Study (UKPDS) Group: Intensive blood-glucose control with sulphonylureas or insulin compared with conventional treatment and risk of complications in patients with type 2 diabetes. Lancet. 1998;352:837-53.

29. Ceriello A, Esposito KE, Piconi L, Ihnat MA, Thorpe JE, Testa R, et al. Oscillating glucose is more deleterious on endothelial function and oxidative stress than mean glucose in normals and type 2 diabetic patients. Diabetes. 2008;57(5):1349-54.

30. Ceriello AA, Morocutti F, Mercuri L, et al. Defective intracellular antioxidant enzyme production in type 1 diabetic patients with nephropathy. Diabetes. 2000;49:2170-7.

31. Hodgkinson AD, Bartlett T, Oates PJ, Millward BA, Demaine AG. The response of antioxidant genes to hyperglycemia is abnormal in patients with type 1 diabetes and diabetic nephropathy. Diabetes. 2003;52:846-851.

32. Scagliusi FB, Lancha Junior AH. Subnotificação da ingestão energética na avaliação do consumo alimentar. Rev Nutr. 2003; 16(4):471-81.

33. Gellar LA, Schrader K, Nansel TR. Healthy eating practices: perceptions, facilitators, and barriers among youth with diabetes. Diabetes Educ. 2007;33(4):671-9.

34. Katz ML,Mehta S, Nansel T, Quinn H, Lipsky LM, Laffel LM. Associations of nutrient intake with glycemic control in youth with type 1 diabetes: differences by insulin regimen. Diabetes Technol Ther. 2014;16(8):512-8.

35. Wolpert HA, Atakov-Castillo A, Smith SA, Steil GM. Dietary fat acutely increases glucose concentrations and insulin requirements in patients with type 1 diabetes: implications for carbohydrate-based bolus dose calculation and intensive diabetes management. Diabetes Care. 2013;36(4):810-6.

36. Marigliano M, Morandi A, Maschio M, Sabbion A, Contreas G, et al. Nutritional education and carbohydrate counting in children with type 1 diabetes treated with continuous subcutaneous insulin infusion: the effects on dietary habits, body composition and glycometabolic control.ActaDiabetol. 2013;50(6):959-64. 
37. Rabasa-Lhoret R, Garon J, Langelier H, Poisson D, Chiasson JL. Effects of meal carbohydrate content on insulin requirements in type 1 diabetic patients treated intensively with the basal-bolus (ultralente-regular) insulin regimen. Diabetes Care. 1999;22(5): $667-73$.

38. Meissner T, Wolf J, Kersting M, Fröheich-Reiterer E, Flechtner-Mors M. Carbohydrate intake in relation to BMI, HbA1c and lipid profile in children and adolescents with type 1 diabetes. Clin Nutr. 2014;33(1):75-8.

39. IV Diretriz Brasileira sobre Dislipidemias e prevenção da aterosclerose Departamento de Aterosclerose da Sociedade Brasileira de Cardiologia. Arq Bras Cardiol. 2007;8(supp 1):2-19.

40. Arcanjo CL, Piccirillo LJ, Machado IV, Andrade Jr CRM, Clemente EL, Gomes MB. Avaliação de dislipidemia e de índices antropométricos em pacientes com diabetes mellitus tipo 1. Arq Bras Endocrinol Metabol. 2005;49(6):951-8.

41. CastroSH, Castro-Faria-Neto HC, Gomes MB. Association of Postprandial Hyperglycemia with in Vitro LDL Oxidation in Non-Smoking Patients with Type 1 Diabetes - a Cross-Sectional Study. Rev Diabet Stud. 2005;2(3):157-64.

42. Queiroz KC, Silva IN, Alfenas RCG. Associação entre fatores nutricionais e o controle glicêmico de crianças e adolescentes com diabetes melito tipo 1. Arq Bras Endocrinol Metab. 2010;54(3):319-25. 